\title{
Induction of Temporal Lobe Epilepsy in Mice with Pilocarpine
}

Muhammad N. Arshad and Janice R. Naegele*

Department of Biology, Program in Neuroscience and Behavior, Wesleyan University, Middletown, CT, 06459, USA

*For correspondence: jnaegele@wesleyan.edu

[Abstract] In the pilocarpine model of temporal lobe epilepsy (TLE) in rodents, systemic injections of pilocarpine induce continuous, prolonged limbic seizures, a condition termed "Status Epilepticus" (SE). With appropriate doses, many inbred strains of mice show behavioral seizures within an hour after pilocarpine is injected. With the behavioral scoring system based on a modification of the original Racine scale, one can monitor the seizures behaviorally, as they develop into more prolonged seizures and SE. SE is typically associated with damage to subsets of hippocampal neurons and other structural changes in the hippocampus and generally subsides on its own. However, more precise control of the duration of SE is commonly achieved by injecting a benzodiazepine into the mouse 1 to $3 \mathrm{~h}$ after the onset of SE to suppress the seizures. Several days following pilocarpine-induced SE, electrographic and behavioral seizures begin to occur spontaneously. The goal of this protocol is to reliably generate mice that develop spontaneous recurrent seizures (SRS) and show the typical neuropathological changes in the brain characteristic of severe human mesial temporal lobe epilepsy (mTLE), without high mortality. To reduce mortality, multiple subthreshold injections of pilocarpine are administered, which increases the percentage of mice developing SE without concomitant mortality. Precise control of the duration of SE (1 or $3 \mathrm{~h}$ ) is achieved by suppressing SE with the benzodiazepine Midazolam (Versed). We have found that this protocol is an efficient means for generating mice that subsequently develop characteristics of human mTLE including high-frequency interictal spike and wave activity and SRS. In addition, we and others have shown that this protocol produces mice that show excitotoxic cell death of subsets of hippocampal GABAergic interneurons, particularly in the dentate gyrus and compensatory sprouting of excitatory projections from dentate granule cells (mossy fiber sprouting). Aspects of this protocol have been described in several of our previous publications.

Keywords: Hippocampus, Seizures, Neurodegeneration, Epileptogenesis, Pilocarpine

[Background] The mouse pilocarpine model of TLE is an experimental paradigm that has been widely used as an animal model of human mTLE. This model has been used for testing long-term efficacy of novel therapies for suppressing SRS and improving cognitive and neuropathological changes associated with severe mTLE. This rodent model of TLE recapitulates many of the neuropathological hallmarks in mTLE, including severe seizures, loss of GABAergic interneurons in the dentate gyrus, Ammon's Horn sclerosis, and cognitive impairments (Swartz et al., 2006). Although some patients with mTLE can be treated successfully anti-convulsant medications, about one-third experience intractable pharmaco-resistant seizures and surgical removal of the seizure foci are required. If the foci are located 
in the mesial temporal lobes, surgical removal may not be feasible due to the role of the hippocampus in memory formation and consolidation. For these patient populations, novel treatments are needed. The cost-effective pilocarpine model of TLE in rodents provides an experimental system for testing novel therapies because a high percentage of rodents develop SRS that can be monitored by video electroencephalography ( $v-E E G$ ) for weeks or months. Novel treatments that suppress seizures can also be studied for their effects on the neuropathological changes observed in this model, including mossy fiber sprouting (Henderson et al., 2014), dispersion of the granule cell layer (Scharfman et al., 2000), and aberrant neurogenesis (Parent et al., 1997).

Pilocarpine hydrochloride (referred to as pilocarpine) is a drug that directly stimulates muscarinic cholinergic receptors in the central nervous system and in the parasympathetic branch of the peripheral nervous system. As a parasympathomimetic drug, pilocarpine induces exocrine gland secretion and stimulates smooth muscle contractions in the gut and secretory glands. It is also a chemoconvulsant that is used to elicit limbic seizures in rodents. Pilocarpine is typically administered by subcutaneous (Walter et al., 2007) or intraperitoneal (i.p.) injections in rodents to induce a condition of continuous generalized seizures, called Status Epilepticus (SE). After the mice develop SE in this model, the experimenter terminates the seizures by injecting a sedative dose of benzodiazepine, a class of drugs that acts on gamma-aminobutyric acid-A (GABA-A) receptors in the brain. Typically, rodents that have experienced one or more hours of SE, will develop TLE with high-frequency interictal spike, wave activity, and SRS (Mello et al., 1993; Wozny et al., 2003; Knopp et al., 2005; Henderson et al., 2014).

In a comprehensive study of the multiple factors contributing to the development of SRS in the rodent pilocarpine model, the strongest correlations were found between dentate gyrus GABAergic neuron loss and seizure frequency (i.e., disease severity) (Buckmaster et al., 2017). Many human TLE patients and rodents subjected to pilocarpine-induce SE show loss of somatostatin-expressing GABAergic interneurons in the hippocampus (Robbins et al., 1991; Borges et al., 2003; Hoffman et al., 2016). In the mouse pilocarpine model, GABAergic interneurons in the hilus of the dentate gyrus and CA1 that coexpress somatostatin and Striatal Enriched Tyrosine Phosphatase (STEP) were found to be highly vulnerable to SE (Buckmaster and Jongen-Relo, 1999; Choi et al., 2007; Zhang et al., 2009). Associated with the loss of GABAergic interneurons, studies reported reduced synaptic inhibition (Kobayashi and Buckmaster, 2003), despite the fact that many of the GABAergic interneurons in the dentate gyrus and CA1 that survive after SE sprout additional inhibitory projections (Wittner et al., 2002; Bausch, 2005; Zhang et al., 2009; Thind et al., 2010). Loss of both somatic and distal dendritic GABAergic inhibition to granule cells occurs shortly after SE, but precedes the development of SRS, suggesting that loss of GABAergic inhibition onto granule cells in the dentate gyrus is an important predictor of whether TLE will develop (Kobayashi and Buckmaster, 2003).

Because of the strong link between loss of GABAergic inhibition and development of TLE, we and other groups have tested whether transplantation of GABAergic progenitors, from either the embryonic mouse forebrain or generated in vitro from human pluripotent stem cells, suppresses pilocarpineinduced TLE (Maisano et al., 2012; Henderson et al., 2014; Anderson et al., 2018). Additionally, recent work shows that this model can be used in combination with molecular and electrophysiological 
approaches, such as retroviral labeling and optogenetics, to evaluate cellular mechanisms responsible for seizure suppression following transplantation (Gupta et al., 2019). The methods detailed in this protocol for induction of SE in both male and female rodents can be adapted to different inbred strains of mice with low mortality; a high incidence of the mice that experience SE will subsequently develop SRS and the typical histopathological hallmarks of TLE (Walter et al., 2007; Henderson et al., 2014; Anderson et al., 2018; Gupta et al., 2019).

In the following protocol, we provide the detailed methods for the successful induction of SE with multiple subthreshold doses of pilocarpine in inbred strains of mice. This method builds upon number of prior studies. In a comparison of 8 inbred strains of mice, Schauwecker and colleagues found significant differences in the susceptibility to pilocarpine, mortality and neuropathological changes after SE (Schauwecker, 2012). Doses ranging between 300 and $400 \mathrm{mg} / \mathrm{kg}$ were reported to induce SE successfully. Higher single doses of pilocarpine were correlated with more successful induction of SE but also increased mortality (Turski et al., 1984; Cavalheiro et al., 1991; Curia et al., 2008; Buckmaster and Haney, 2012; Kelly and Coulter, 2017). In contrast, multiple subthreshold doses of pilocarpine were effective for inducing SE and SRS, with much lower mortality rates (Glien et al., 2001; Groticke et al., 2007).

\section{Materials and Reagents}

1. Sterile disposable bottle top filter $(0.2 \mu \mathrm{m})$ (Thermo Scientific, catalog number: 5680020$)$

2. $27 \mathrm{G}$ needles (BD Biosciences, catalog number: 305109)

3. 1cc syringes (BD Biosciences, Luer-Lok tip, catalog number: 309628)

4. Pipette tips (Axygen, catalog number: 14-222-721)

5. Puralube Vet Ophthalmic Ointment (Dechra, catalog number: 17033-211-38)

6. Eppendorf tubes (VWR, catalog number: 89000-030)

7. Cotton tipped applicators (Thomas Scientific, catalog number: 1213Q08)

8. Nesting square (Ancare, catalog number: NES3600)

9. Paper house (Shepherd specialty papers, catalog number: Shepherd shack reg)

10. Lab markers (Sharpie, Amazon, Permanent ultrafine black 12 count)

11. C57BI/6NHsd mice (Envigo, catalog number: 47)

12. Isothesia (Isoflurane) (Henry Schein, catalog number: SKU 029405)

13. $2 \%$ topical lidocaine ( $\mathrm{Hi}$ Tech, catalog number: 00603-1393-64)

14. Povidone iodine (CVS, catalog number: 955338)

15. Sodium chloride ( $\mathrm{NaCl}$ ) (Sigma, catalog number: $\mathrm{S} 9888-5 \mathrm{KG}$ )

16. Scopolamine methyl bromide (Sigma, catalog number: S85002), $4^{\circ} \mathrm{C}$, dispose 9 months after opening, $4{ }^{\circ} \mathrm{C}$

17. Pilocarpine hydrochloride (Sigma, catalog number: P6503-SG), dispose 2 months after opening, $4{ }^{\circ} \mathrm{C}$ 
18. Midazolam (Henry Schein, catalog number: 17478052410), dispose 2 months after opening, store at RT

19. Sterile lactated ringers (Henry Schein, catalog number: 065888 ), store at RT

20. Square container (Rubbermaid, catalog number: 1776401 1-1/4-Cup square container)

21. $0.9 \%$ Saline solution (see Recipes)

22. Scopolamine stock solution (see Recipes)

23. Scopolamine working solution (see Recipes)

24. Pilocarpine stock solution (see Recipes)

\section{Equipment}

1. Calculator (Casio, catalog number: FX-991EX)

2. P1000 pipette (Eppendorf, catalog number: 2231000601)

3. P200 pipette (Eppendorf, catalog number: 2231000601)

4. Weighing balance (Metler, catalog number: AE 240)

5. Vortex (Fisher Scientific, Genie 2, catalog number: 12-812)

6. Heating pad (Sunbeam, catalog number: 731-500)

7. Barnstead Nanopure (Thermo Fisher Scientific, catalog number: D11921)

8. Silver wire (World Precision Instruments, catalog number: AGW1030)

9. Six-pin connectors (Allied Electronics, catalog number: R1023090

10. Stainless steel screws (Plastics One, catalog number: 00-96×3-32)

11. Silver print (GC Electronics, catalog number: 22-023)

12. Dental acrylic cement (Lang Dental Manufacturing, catalog number: 0830 Fibred Pink)

13. Timer (Fisher Scientific, catalog number: 14-648-17)

14. Preamplifier (Pinnacle Technology, catalog number: 8406-SE4)

15. Electrical commutator (Pinnacle Technology, catalog number: 8408)

16. Data acquisition device (Pinnacle Technology, catalog number: 8401)

17. Automated stereotaxic injection system (Stoelting Quintessential, catalog number: 53311)

18. Charcoal filter (VetEquip, catalog number: 931401)

19. Dissecting microscope (Zeiss OPMI 6, catalog number: 93088)

\section{Software}

1. Sirenia Seizure Pro Software, including Sirenia Acquisition and Sirenia Seizure Detection $\begin{array}{lllll}\text { modules } & \text { (Pinnacle } & \text { Technology, } & \text { software }\end{array}$ https://www.pinnaclet.com/seizurePRO.html) 


\section{Procedure}

A. Mouse ordering, housing and handling

1. 3-4-week-old C57BI/6 mice from Envigo or Jackson Labs.

2. House the mice in individual cages with a single nesting square and a paper house for 1-2 weeks before experimentation on a 12-h light/dark cycle.

3. Give free access to food and water. Weigh mice at 5-6 weeks of age. Handle the mice daily for $\sim 5$ min for a few days prior to SE induction, as handling appears to reduce the stress response. Note: This protocol works well in mice weighing between 18-22 $\mathrm{g}$ and is suitable for both male and female C57BI/6N mice from (Envigo) as well as some transgenic inbred strains from Jackson Labs (e.g., NSG). Research personnel should be well-trained in procedures for safe and appropriate handling and restraint of mice when administering substances. The use of animal models of epilepsy should be guided by the goals of the research, while also giving priority to animal welfare and effective animal treatment and care (Lidster et al., 2016).

B. Day of seizure induction

Induce SE between 08:00 A.M. and 12:00 P.M. during the light portion of the dark/light cycle (07:00 A.M. to 07:00 P.M.) to control for diurnal variations (Walter et al., 2007; Henderson et al., 2014; Anderson et al., 2018; Gupta et al., 2019). The optimal dose of pilocarpine is critical for successful SE induction in mice and should be worked out based on the strain used in the study.

To induce SE in mice, first inject scopolamine $(0.5 \mathrm{mg} / \mathrm{ml}$, i.p.), followed by an injection of pilocarpine $(280 \mathrm{mg} / \mathrm{kg}$; i.p.). If the mouse fails to develop seizure in $30 \mathrm{~min}$ after the first injection, 1 or 2 supplemental doses of pilocarpine $30-60 \mathrm{mg} / \mathrm{kg}$, i.p. can be administered to induce SE. Mice injected with pilocarpine at these doses will generally develop generalized motor seizures that may include jumping and running. During induction of SE, the experimenter monitors behavioral seizures visually, based on a modification of a behavioral scoring system originally developed by Racine (Racine, 1972). In the modified version, the severity of behavioral seizures are scored (Shibley and Smith, 2002) (see Table 1). Mice that develop SE exhibit repetitive head bobbing and a lack of motor control that continues after the seizures are attenuated with Midazolam. The rhythmic head movements typically subside after $\sim 6 \mathrm{~h}$ but body tics or brief limb/body twitches may continue. 
Table 1. Modified Racine scale for scoring seizures

\begin{tabular}{ll}
\hline Exhibited Behavior & Scale Score \\
\hline Normal Activity & 0 \\
Immobilization (frozen posture) & 1 \\
Facial automatisms (whisker twitching, opening and closing mouth) & 2 \\
Stiffened/extended tail (Straub) & 2.5 \\
Partial body clonus-forelimb or hindlimb & 3 \\
Rearing & 4 \\
Tonic-clonic w/ loss of posture (mouse on its side) & $\mathbf{5}$ \\
\hline
\end{tabular}

Monitoring and scoring pilocarpine-induced seizures and SE: The modified Racine Scale shown in Table 1 is used during pilocarpine induction of SE to observe and classify different levels of seizures (Shibley and Smith, 2002). The original scale included 5 behavior categories of seizures. In the pilocarpine model in mice, several stages may occur together in rapid succession, making it difficult to distinguish stage 4 . This rating system is convenient, but less sensitive than EEG detection of seizures. The following video recordings illustrate three stages of seizures, stage 3 (Video 1), stage 5 (Video 2), and SE (Video 3).

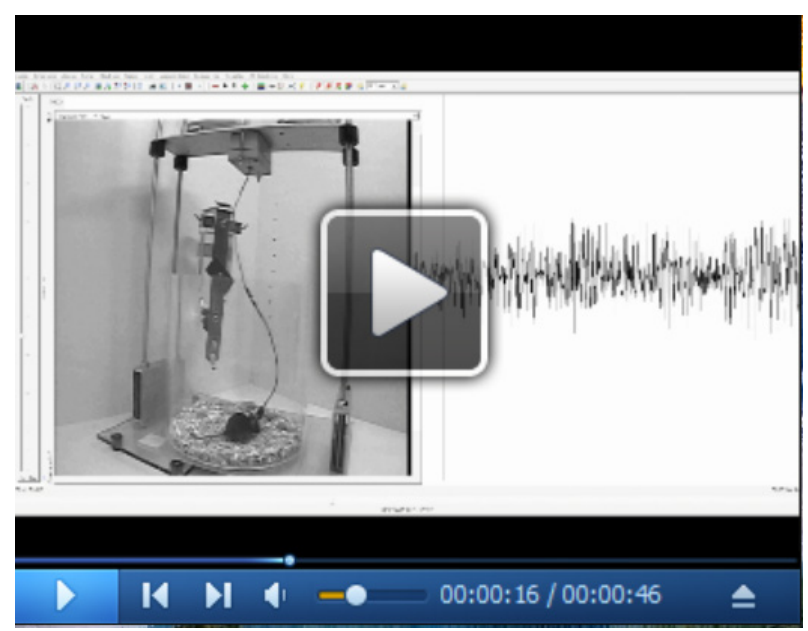

Video 1. Video-EEG of a mouse exhibiting Modified Racine Stage 3 seizures. In this stage, pilocarpine-treated mice develop generalized tonic-clonic seizures associated with a stiff tail, involuntary movements of the forelimbs, and orofacial movements of mouth and whiskers (Video 1). The behavioral seizure begins at $14 \mathrm{~s}$ in the video. 


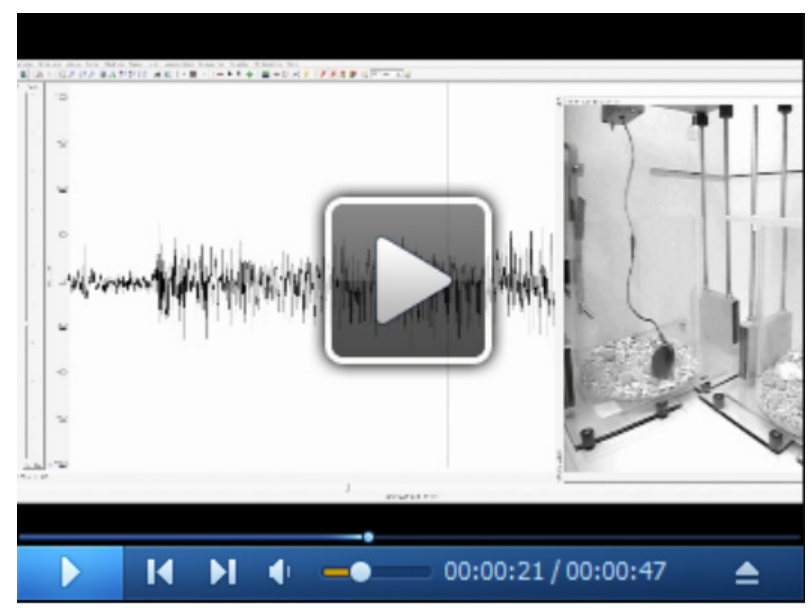

Video 2. Video-EEG of a mouse exhibiting Modified Racine Scale Stage 5 seizures. The seizure begins at $22 \mathrm{~s}$ in this video and shows the characteristic behaviors of mice in Stage 5 seizures. These include rearing up onto hind legs and falling, as well as involuntary, bilateral, tonic-clonic movements of the limbs. In this video, the mouse briefly loses postural control and then regains it, which is also typical.

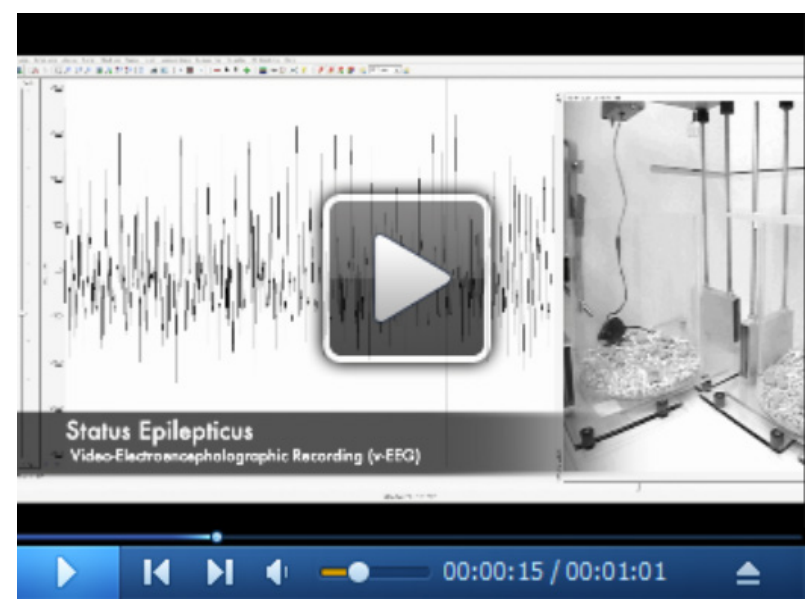

Video 3. Video-EEG of a mouse exhibiting SE. In this stage called SE, mice have experienced 3 or more stage 3-5 seizures and develop continuous seizures, or SE. In this stage, the mice become immobile and exhibit prominent head-tics.

\section{Before starting seizure induction}

1. Prepare scopolamine stock solution, scopolamine working solution, and the pilocarpine working solution, as detailed in Recipes. Prepare these solutions on the day of SE induction and discard after using.

2. Put all the liquid reagents (saline, scopolamine, pilocarpine) on ice.

3. Bring reagents, $0.5 \mathrm{ml}$ Eppendorf tubes, calculator, lab markers, pipette, and pipette tips up to the procedure room to induce SE.

4. Approximately 15 mice are induced in the same session. Typically, 4 trained observers are needed to monitor seizures for 15 mice. Each observer then monitors 3 or 4 mice. 
5. Weigh the mice individually in a square container with a lid (Rubbermaid container).

6. Remove extra bedding, food, water, and environmental enrichment from the cages prior to seizure induction.

7. Label each cage with the experimental number of the mouse, label the Eppendorf tubes that will contain the injectables for that mouse, and the disposable syringe for that mouse.

8. Prepare the pilocarpine working solutions for each mouse (calculations are provided below).

9. Calculations for pilocarpine working solution:

a. Prepare the Eppendorf tube for each mouse by diluting pilocarpine stock solution with $0.9 \%$ sterile saline, in a final volume of $300 \mu \mathrm{l}$.

b. The volume of pilocarpine stock solution should be calculated as follows:

$6 \times($ mouse weight $)=X$ pilocarpine $(\mu \mathrm{l})$

c. The volume of $0.9 \%$ sterile saline is calculated as follows:

$300 \mu \mathrm{l}$ minus volume of pilocarpine stock $(\mu \mathrm{l})$

Example: 21.2-gram mouse

Volume of Pilocarpine $(\mu \mathrm{l}): 6$ × $21.2(\mathrm{~g})=127.2$

$0.9 \%$ sterile saline: $300 \mu \mathrm{l}-127.2 \mu \mathrm{l}=172.8 \mu \mathrm{l}$

\section{SE induction procedure}

1. Inject each mouse with $0.07 \mathrm{ml}$ scopolamine $(\sim 0.5 \mathrm{mg} / \mathrm{ml}$; i.p.) and note the time of injection.

2. $30 \mathrm{~min}$ after the scopolamine injection, inject the working pilocarpine solution $(0.1 \mathrm{ml}$; i.p.). Note that the dilution is specific for each mouse (see "Before starting seizure induction" of Section B, Step 9).

3. Note the time of each seizure event and the duration (count motor seizures lasting $15 \mathrm{~s}$ or longer only).

4. If mouse does not exhibit any stage 4 or stage 5 seizures within 20-30 min of the first injection, inject a supplemental dose of pilocarpine (0.01-0.02 ml, i.p.) using the solution prepared for that mouse. If the mouse has one or more stage 3 seizures, but no stage 4 or 5 seizures, the supplemental dose is $0.01 \mathrm{ml}$. However, if a mouse exhibits stage 0,1 or 2 but no stage 3,4 , or 5 seizures, the mouse is given a supplemental dose of $0.02 \mathrm{ml}$ (i.p.).

5. After 3-5 times stage 3, 4, or 5 Racine scale seizures, the mouse will typically develop SE, defined as a continuous state of seizures characterized by a prominent head tic or "hiccup", rigidity (body and tail), and immobility. Note the time of SE.

6. SE will typically continue unabated. One to $3 \mathrm{~h}$ after the time of the initial onset of SE, inject $0.04 \mathrm{ml}$ Midazolam (i.p.) to attenuate the seizures (Figure 1). Gently scruff mouse and apply ophthalmic ointment with cotton swab to each eye to prevent the corneas from drying out, as mice that develop SE blink infrequently.

7. Monitor each mouse in its home cage for $6 \mathrm{~h}$ following attenuation of SE with midazolam. Provide additional warmth for several hours using a heating pad under the cage, until the mouse is walking and drinking. 
8. Provide a nesting square, a paper house, food and water ad libitum, and maintain the mice in the 12-h light/12-h dark cycle. We routinely provide all mice with moistened/softened food for several days, as mice may become constipated following induction of SE.

9. Monitor the mice and administer Ringer's solution daily for several days (1 ml, i.p.). Inject $2 x /$ day only when a mouse becomes immobile or fails to eat or groom.

Notes:

a. One hour of SE is sufficient for generating mice that reliably develop TLE with recurrent spontaneous seizures. In our laboratory, the typical success rate for inducing SE with this method is $\sim 70 \%$ or higher. Three hours of SE results in a more severe disease phenotype, but longer periods of SE are associated with increased mortality rates.

b. Animal welfare assessments must be conducted multiple times per day for several days after the induction of SE. The mice should be provided ad libitum with softened food and hydrated with sterile Ringer's lactate injections (i.p.) for several days after induction of SE. Mice may lose weight and show increased aggression after SE, necessitating individual housing. If a mouse becomes immobile or fails to eat or groom for more than $24 \mathrm{~h}$, the mouse should be euthanized. Mortality and suffering of animals can be minimized by ensuring that the same experimenter handles the mice and by avoiding unnecessary loud noises and startling of the animals. Additional considerations regarding use of rodents in epilepsy research models may be found on the web at the National Centre for the Replacement, Refinement, and Reduction of Animals in Research (NC3Rs). 
Inject methyl scopolamine

Inject pilocarpine to induce seizures (30 min after methyl scopolamine)

Visually score motor seizures and inject supplemental doses of pilocarpine, if needed

Mice develop status epilepticus, lasting 60-180 min

Inject midazolam to attenuate seizures

Figure 1. Flow chart diagram of experimental protocol for inducing status epilepticus

C. Electrode implantation

1. Prepare the mouse for surgery by anesthetizing with isoflurane gas in an induction chamber. Confirm that the animal is properly anesthetized by pinching foot.

2. Place the mouse in the stereotaxic apparatus. Line up the front incisors in the opening in nose clamp and fix the nose cone in place to deliver isoflurane gas anesthesia during surgery.

3. Adjust the ear bars, making sure that they immobilize the mouse's skull, without excessive pressure.

4. Apply ophthalmic ointment to the mouse's eyes with a sterile cotton swab.

5. Sterilize the skin by applying povidone-iodine and lidocaine with sterile cotton swab, to reduce pain.

6. Make a longitudinal incision along the midline axis of the skull with the scalpel blade, exposing bregma and lambda and gently push aside muscles that are attached to the top of the skull.

7. Apply $\mathrm{H}_{2} \mathrm{O}_{2}$ to the skull with a sterile cotton swab to aid in bregma and lambda visualization through dissecting microscope.

8. For surgical implantation of subdural electrodes, drill two midline holes in the skull; one anterior to bregma and one posterior to lambda. Drill four additional holes (two per side) with reference to bregma, at coordinates AP: $-1.5 \mathrm{~mm}$; L: $\pm 2.5 \mathrm{~mm}$ and AP: $-3.0 \mathrm{~mm}$; L: $\pm 2.5 \mathrm{~mm}$. 
9. The electrode head mount consists of $0.25 \mathrm{~mm}$ silver wires soldered to six-pin connectors. The ends of the four subdural electrode silver wires are heated to form smooth balls at the ends, to avoid damage to the cortex by the wires.

10. Insert stainless steel screws into the two holes in the skull that are anterior to bregma and posterior to lambda. Wrap the two electrode anchor silver wires serving as reference and ground connections around these screws.

11. Apply silver print to these connections to ensure good electrical contacts.

12. Insert the four subdural electrode wires into the holes in the skull positioned at coordinates AP: $-1.5 \mathrm{~mm}$; L: $\pm 2.5 \mathrm{~mm}$ and AP: $-3.0 \mathrm{~mm} ; \mathrm{L}: \pm 2.5 \mathrm{~mm}$, with reference to the bregma. Make certain that the balls at the ends of the electrode wires are in light contact with the surface of the brain.

13. Apply dental acrylic cement to cover and hold the electrode head mount in place.

14. Remove the mouse from the stereotaxic apparatus and place it in a clean cage on a heating pad. Monitor the mouse until it regains consciousness and begins to move around. Return the mouse to the home cage.

D. Data acquisition and scoring of SRS by Video-EEG following SE induction

The pilocarpine model is often used as a chronic model of TLE because the mice will begin to spontaneous seizures, typically occurring in clusters of seizures that last 4-5 days with multiple brief seizures/day, and seizure-free intervals that can last for periods of days or even weeks. Although the onset of SRS varies, we find it useful to begin to monitor 3 weeks after SE induction because the mice begin to exhibit more frequent seizures by 3-4 weeks. The effects of a particular experimental treatment on seizures can be accurately monitored using video (V)-EEG recordings (Curia et al., 2008). We typically conduct continuous v-EEG recordings for 3 weeks or longer, when assessing the efficacy of a particular therapeutic treatment.

When performing multiple stereotaxic surgeries, we typically wait at least 1 week after SE. In our prior work, we made stereotaxic injections of stem cells at $\sim 2$ weeks after SE and stereotaxic injections of retrovirus at 1 week before, or after, stem cell transplantation. We typically have an experimental treatment group (e.g., mice that receive stem cell transplants) and compare them to a control group that receives vehicle (or stereotaxic injections of the media minus stem cells). We then implant subdural electrodes for chronic v-EEG recordings at $\sim 3$ weeks after SE. To detect and quantify SRS, it is useful to carry out continuous v-EEG recordings for 3 weeks or longer. For v-EEG recordings, EEG signals are amplified with a preamplifier connected to the data acquisition device and recorded with Sirenia Seizure Pro software (Pinnacle Technology), an analysis package for quickly identifying and analyzing user-defined seizure events based on power and line length. Data are captured using Sirenia Acquisition software at $1,000 \mathrm{~Hz}$ with a gain of $10 \mathrm{x}$, using high and low pass-filters set at $0.5 \mathrm{~Hz}$ and $100 \mathrm{~Hz}$ respectively. Video recordings are synchronized with EEG signals by the Sirenia seizure monitoring software. We use changes in power and line length deviations from baseline EEG brain activity to detect seizures in this software (Henderson et al., 2014; Anderson et al., 2018). After detecting potential seizures with the software, each of the seizure 
events should be verified manually by an experimenter who views the EEG and associated video recording and scores the severity of the seizures based on the modified Racine scale (Table 1; Figure 2). Verification is necessary to distinguish true seizures from occasional EEG artifacts caused by grooming or other issues with the head-mounted electrodes.

1. Open "Sirenia Seizure". Click "File", then select the file to analyze.

2. Click "Time Span" and choose "1 Minute". Click "Time Step" and choose "10 s".

3. Right-click on the y-axis tab to the left of each EEG channel and click "Manual".

4. Click in the text boxes that appear on the top and bottom of each y-axis tab. Change the top box to "500" and the bottom box to "-500" to scale down the EEG trace for easier viewing of seizure activity. Seizure activity is usually about $\pm 500 \mu \mathrm{V}$ or more than twice baseline amplitude, for each mouse.

5. Click the "Seizure Settings" icon. Change the "Window Size" and "Step Size" to 5.00.

6. Select the "Line Length" search option and adjust the threshold to 6,000.

7. Make sure all EEG channels are selected under "Use Channels".

8. Click the "Next Possible Seizure" button to begin searching for seizures.

9. The "Search Progress" window will open. When a seizure is found, the search will pause, and the region of activity detected as greater than the specified line length in the window size and step will be highlighted in pink.

10. Use the "Pan" tool to identify the beginning and the end of the seizure event.

11. Begin $\sim 10$ s prior to detected seizures and click the "Play" icon to start the playback for scoring behavioral seizures.

12. Watch the camera recording to score the mouse's behavior.

13. EEG 1 and 2 in the Sirenia seizure software measure the EEG activity in the right and left hemisphere respectively of the mouse in the camera's view. However, EEG 3 is a reference electrode. See Figure 3.

14. Click the "Next Possible Seizure" icon to begin searching for the next seizure. 
A Example of baseline EEG activity (1 minute window)

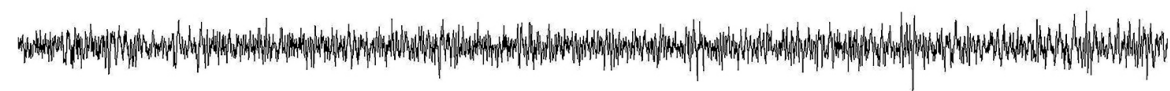

B Example of an abnormal spiking activity (1 minute window) $\prod_{3 \sec }^{200 \mu \mathrm{v}}$

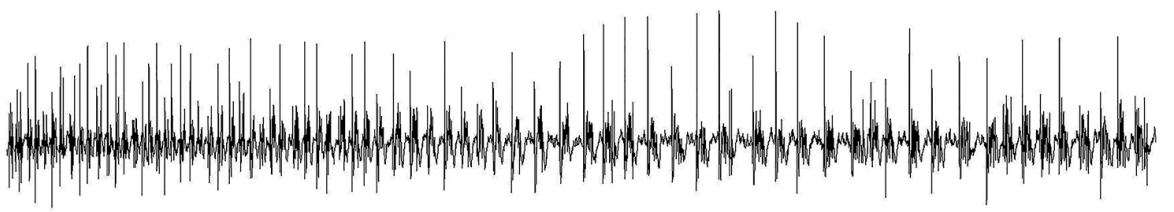

C Example of a seizure activity (1 minute window)

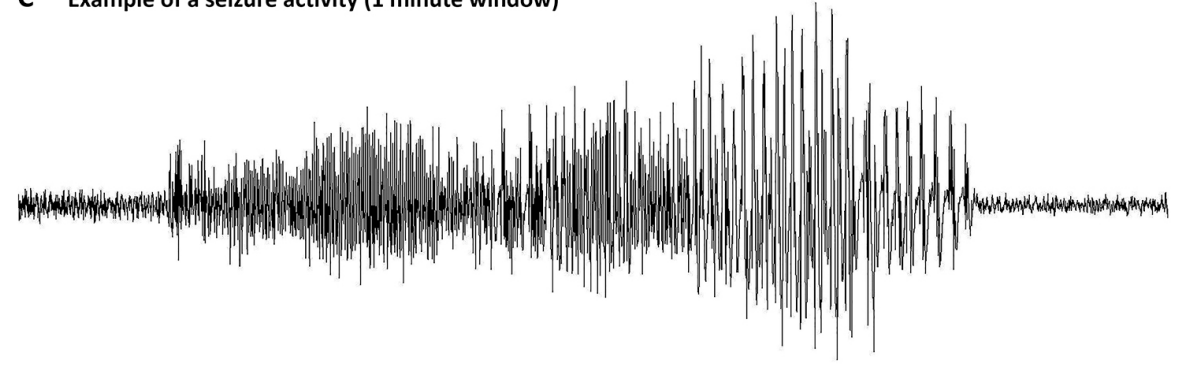

D Example of Status Epilepticus EEG trace (1 minute window)

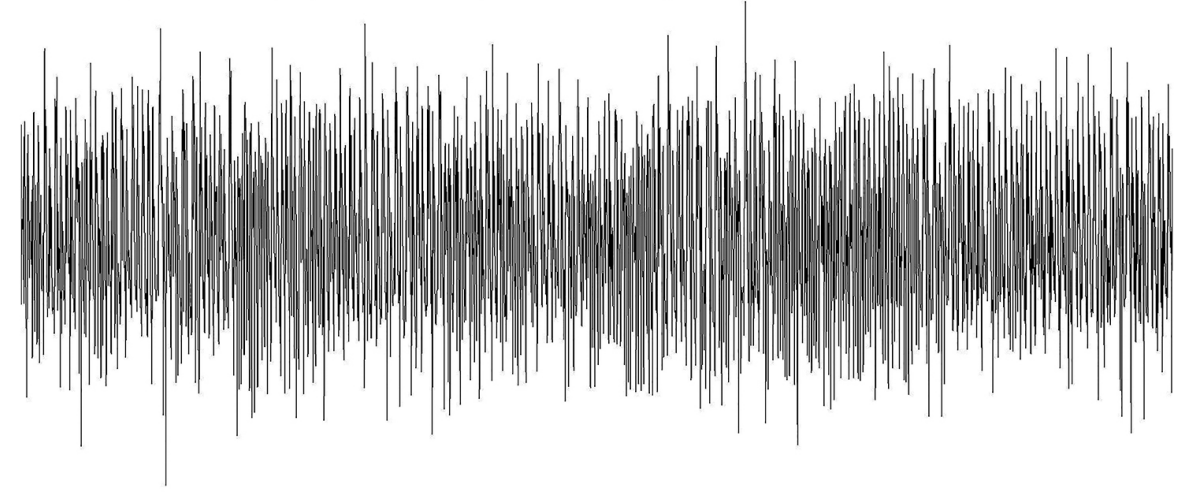

Figure 2. Examples of spontaneous epileptiform activity in mice following pilocarpineinduced SE. The EEGs were acquired using Sirenia Acquisition and Sirenia Seizure Detection software. 


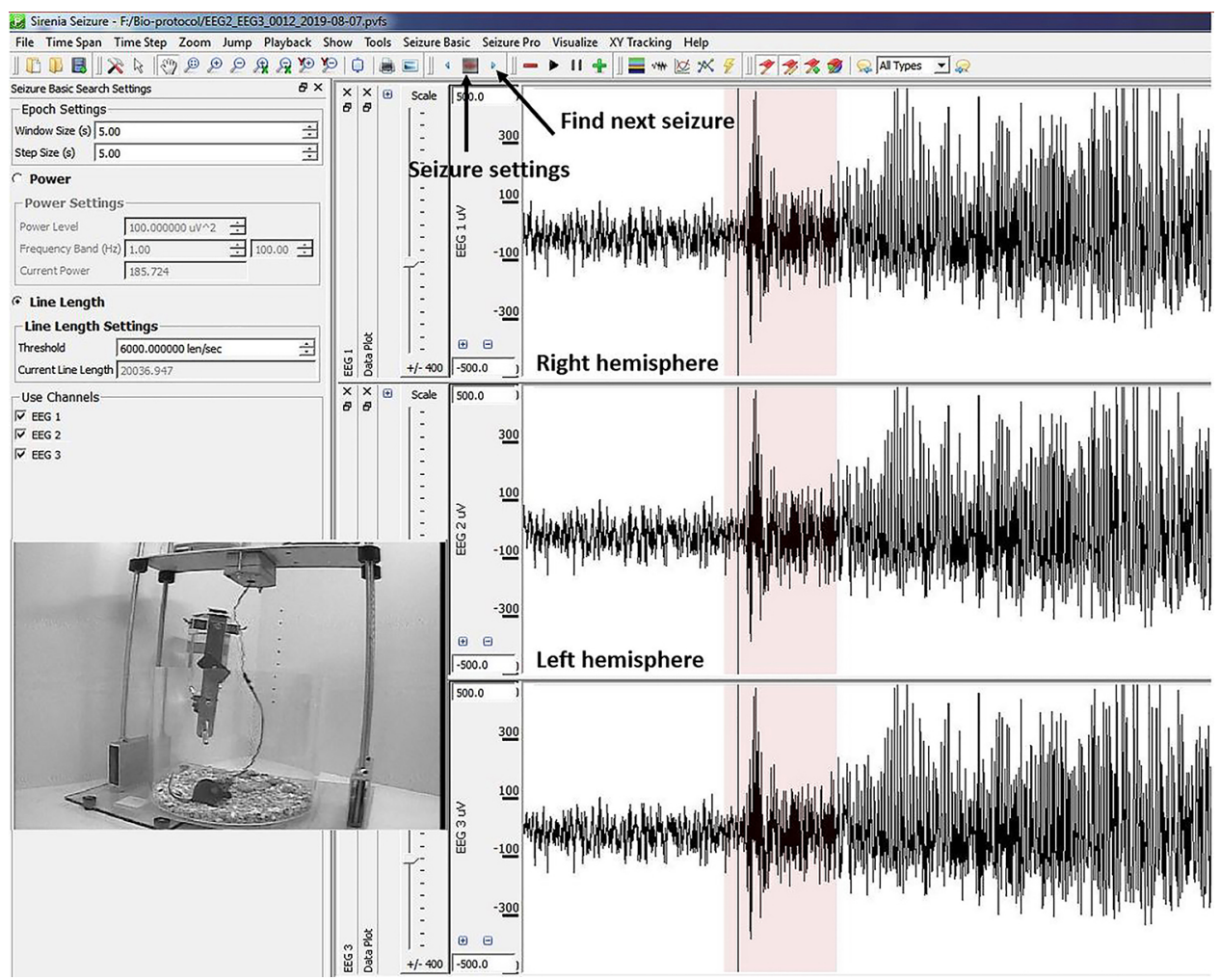

Figure 3. The graphical user interface in the Sirenia seizure detection software. The setting for detection of seizures is based on the EEG line length, with a threshold setting of 6000.000 len/sec. The mouse is shown in a video camera view that has been superimposed onto the screenshot of the graphical user interface.

\section{$\underline{\text { Recipes }}$}

1. $0.9 \%$ sterile saline solution

$\begin{array}{ll}\text { Reagent } & \text { Amount: } 500 \mathrm{ml} \\ \mathrm{NaCl} & 4.5 \mathrm{~g}\end{array}$

Add $500 \mathrm{ml}$ MilliQdd $_{2} \mathrm{O}$

Sterilize the solution by passing through a $0.2 \mu \mathrm{m}$ bottle top filter

2. Scopolamine stock solution
Reagent
Amount: $1 \mathrm{ml}$
Scopolamine Methyl Bromide
$10 \mathrm{mg}$

Dissolve scopolamine in $998 \mu \mathrm{l}$ of $0.9 \%$ sterile saline, for a final volume of $1 \mathrm{ml}$

3. Scopolamine working solution

Reagent

Scopolamine stock solution

$0.9 \%$ sterile saline

This amount is sufficient for 8 mice

4. Pilocarpine stock solution
Amount: $1 \mathrm{ml}$

$950 \mu \mathrm{l}$

$50 \mu \mathrm{l}$ 


$\begin{array}{lc}\text { Reagent } & \text { Amount: } 1 \mathrm{ml} \\ \text { Pilocarpine } \mathrm{HCl} & 140 \mathrm{mg} \\ 0.9 \% \text { sterile saline } & \text { to } 1 \mathrm{ml}\end{array}$

Dissolve pilocarpine in $888 \mu \mathrm{l}$ of $0.9 \%$ sterile saline, for a final volume of $1 \mathrm{ml}$

\section{Acknowledgments}

This work was funded by grants from National Institutes of Health (NINDS grant R15NS07287901A1), McKnight Foundation Brain Disorders Award (JRN), A Challenge Award from Citizens United for Research in Epilepsy (JRN), The Regenerative Medicine Research Fund of Connecticut Established Investigator Grant (JRN), and the Alan M. Dachs Endowed Professorship at Wesleyan University (JRN). We also thank our colleagues and collaborators who contributed to published work that was based on variations of this protocol: Bryan Luikart, Karl Obrietan, Yun-Sik Choi, Paul Lombroso, Laura Grabel, and Gloster Aaron. We thank lab members Jaye Jeong and Nicolas Cimino for contributing to discussions about this manuscript. We thank former lab technicians who helped us develop this detailed protocol: Sara Royston, Stephanie Tagliatela, Katharine Henderson, Dan Lawrence, and Kevin Cobbol.

\section{Competing interests}

The authors have no competing interests.

\section{Ethics}

Wesleyan University Institutional Animal Care and Use Committee confirms that this protocol (Protocol \# 2017_0531_Naegele(C), Project; valid through 05/30/20) conforms to all Federal and State laws and guidelines and all institutional policies and procedures concerning the care and use of animal in research, teaching, and testing.

\section{References}

1. Anderson, N. C., Van Zandt, M. A., Shrestha, S., Lawrence, D. B., Gupta, J., Chen, C. Y., Harrsch, F. A., Boyi, T., Dundes, C. E., Aaron, G., Naegele, J. R. and Grabel, L. (2018). Pluripotent stem cell-derived interneuron progenitors mature and restore memory deficits but do not suppress seizures in the epileptic mouse brain. Stem Cell Res 33: 83-94.

2. Bausch, S. B. (2005). Axonal sprouting of GABAergic interneurons in temporal lobe epilepsy. Epilepsy Behav 7: 390-400. 
3. Borges, K., Gearing, M., McDermott, D. L., Smith, A. B., Almonte, A. G., Wainer, B. H. and Dingledine, R. (2003). Neuronal and glial pathological changes during epileptogenesis in the mouse pilocarpine model. Exp Neurol 182(1): 21-34.

4. Buckmaster, P. S. and Jongen-Relo, A. L. (1999). Highly specific neuron loss preserves lateral inhibitory circuits in the dentate gyrus of kainate-induced epileptic rats. J Neurosci 19(21): 95199529.

5. Buckmaster, P. S., Abrams, E. and Wen, X. (2017). Seizure frequency correlates with loss of dentate gyrus GABAergic neurons in a mouse model of temporal lobe epilepsy. $J$ Comp Neurol 525(11): 2592-2610.

6. Buckmaster, P. S. and Haney, M. M. (2012). Factors affecting outcomes of pilocarpine treatment in a mouse model of temporal lobe epilepsy. Epilepsy Res 102(3): 153-159.

7. Cavalheiro, E. A., Leite, J. P., Bortolotto, Z. A., Turski, W. A., Ikonomidou, C. and Turski, L. (1991). Long-term effects of pilocarpine in rats: structural damage of the brain triggers kindling and spontaneous recurrent seizures. Epilepsia 32(6): 778-782.

8. Choi, Y. S., Lin, S. L., Lee, B., Kurup, P., Cho, H. Y., Naegele, J. R., Lombroso, P. J. and Obrietan, K. (2007). Status epilepticus-induced somatostatinergic hilar interneuron degeneration is regulated by striatal enriched protein tyrosine phosphatase. J Neurosci 27(11): 2999-3009.

9. Curia, G., Longo, D., Biagini, G., Jones, R. S. and Avoli, M. (2008). The pilocarpine model of temporal lobe epilepsy. J Neurosci Methods 172(2): 143-157.

10. Glien, M., Brandt, C., Potschka, H., Voigt, H., Ebert, U. and Loscher, W. (2001). Repeated lowdose treatment of rats with pilocarpine: low mortality but high proportion of rats developing epilepsy. Epilepsy Res 46(2): 111-119.

11. Groticke, I., Hoffmann, K. and Loscher, W. (2007). Behavioral alterations in the pilocarpine model of temporal lobe epilepsy in mice. Exp Neurol 207(2): 329-349.

12. Gupta, J., Bromwich, M., Radell, J., Arshad, M. N., Gonzalez, S., Luikart, B. W., Aaron, G. B. and Naegele, J. R. (2019). Restrained dendritic growth of adult-born granule cells innervated by transplanted fetal GABAergic interneurons in mice with temporal lobe epilepsy. eNeuro 6(2): pii: ENEURO.0110-18.2019.

13. Henderson, K. W., Gupta, J., Tagliatela, S., Litvina, E., Zheng, X., Van Zandt, M. A., Woods, N., Grund, E., Lin, D., Royston, S., Yanagawa, Y., Aaron, G. B. and Naegele, J. R. (2014). Longterm seizure suppression and optogenetic analyses of synaptic connectivity in epileptic mice with hippocampal grafts of GABAergic interneurons. J Neurosci 34(40): 13492-13504.

14. Kelly, M. E., and Coulter, D. A. (2017). Chapter 42: The pilocarpine model of acquired epilepsy. In: Models of Seizures and Epilepsy. 2nd Edition. Pitkänen, A., Buckmaster, P. S., Galanopoulou, A. S., and Moshé, L. S. (Eds.). London, UK: Academic Press, 625-636.

15. Knopp, A., Kivi, A., Wozny, C., Heinemann, U. and Behr, J. (2005). Cellular and network properties of the subiculum in the pilocarpine model of temporal lobe epilepsy. $J$ Comp Neurol 483(4): 476-488. 
Please cite this article as: Arshad and Naegele, (2020). Induction of Temporal Lobe Epilepsy in Mice with Pilocarpine,Bio-protocol 10 (4): e3533. DOI:

16. Kobayashi, M. and Buckmaster, P. S. (2003). Reduced inhibition of dentate granule cells in a model of temporal lobe epilepsy. $J$ Neurosci 23(6): 2440-2452.

17. Lidster, K., Jefferys, J. G., Blumcke, I., Crunelli, V., Flecknell, P., Frenguelli, B. G., Gray, W. P., Kaminski, R., Pitkanen, A., Ragan, I., Shah, M., Simonato, M., Trevelyan, A., Volk, H., Walker, M., Yates, N. and Prescott, M. J. (2016). Opportunities for improving animal welfare in rodent models of epilepsy and seizures. $J$ Neurosci Methods 260: 2-25.

18. Maisano, X., Litvina, E., Tagliatela, S., Aaron, G. B., Grabel, L. B. and Naegele, J. R. (2012). Differentiation and functional incorporation of embryonic stem cell-derived GABAergic interneurons in the dentate gyrus of mice with temporal lobe epilepsy. $J$ Neurosci 32(1): 46-61.

19. Mello, L. E., Cavalheiro, E. A., Tan, A. M., Kupfer, W. R., Pretorius, J. K., Babb, T. L. and Finch, D. M. (1993). Circuit mechanisms of seizures in the pilocarpine model of chronic epilepsy: cell loss and mossy fiber sprouting. Epilepsia 34(6): 985-995.

20. Parent, J. M., Yu, T. W., Leibowitz, R. T., Geschwind, D. H., Sloviter, R. S. and Lowenstein, D. H. (1997). Dentate granule cell neurogenesis is increased by seizures and contributes to aberrant network reorganization in the adult rat hippocampus. $J$ Neurosci 17(10): 3727-3738.

21. Robbins, R. J., Brines, M. L., Kim, J. H., Adrian, T., de Lanerolle, N., Welsh, S. and Spencer, D. D. (1991). A selective loss of somatostatin in the hippocampus of patients with temporal lobe epilepsy. Ann Neurol 29(3): 325-332.

22. Scharfman, H. E., Goodman, J. H. and Sollas, A. L. (2000). Granule-like neurons at the hilar/CA3 border after status epilepticus and their synchrony with area CA3 pyramidal cells: functional implications of seizure-induced neurogenesis. J Neurosci 20(16): 6144-6158.

23. Schauwecker, P. E. (2012). Strain differences in seizure-induced cell death following pilocarpine-induced status epilepticus. Neurobiol Dis 45(1): 297-304.

24. Shibley, H. and Smith, B. N. (2002). Pilocarpine-induced status epilepticus results in mossy fiber sprouting and spontaneous seizures in C57BL/6 and CD-1 mice. Epilepsy Res 49(2): 109-120.

25. Swartz, B. E., Houser, C. R., Tomiyasu, U., Walsh, G. O., DeSalles, A., Rich, J. R. and DelgadoEscueta, A. (2006). Hippocampal cell loss in posttraumatic human epilepsy. Epilepsia 47(8): 1373-1382.

26. Thind, K. K., Yamawaki, R., Phanwar, I., Zhang, G., Wen, X. and Buckmaster, P. S. (2010).Initial loss but later excess of GABAergic synapses with dentate granule cells in a rat model of temporal lobe epilepsy. J Comp Neurol 518(5): 647-667.

27. Turski, W. A., Cavalheiro, E. A., Bortolotto, Z. A., Mello, L. M., Schwarz, M. and Turski, L. (1984). Seizures produced by pilocarpine in mice: a behavioral, electroencephalographic and morphological analysis. Brain Res 321(2): 237-253.

28. Walter, C., Murphy, B. L., Pun, R. Y., Spieles-Engemann, A. L. and Danzer, S. C. (2007). Pilocarpine-induced seizures cause selective time-dependent changes to adult-generated hippocampal dentate granule cells. J Neurosci 27(28): 7541-7552. 
29. Wittner, L., Eross, L., Szabo, Z., Toth, S., Czirjak, S., Halasz, P., Freund, T. F. and Magloczky, Z. S. (2002). Synaptic reorganization of calbindin-positive neurons in the human hippocampal CA1 region in temporal lobe epilepsy. Neuroscience 115(3): 961-978.

30. Wozny, C., Kivi, A., Lehmann, T. N., Dehnicke, C., Heinemann, U. and Behr, J. (2003). Comment on "On the origin of interictal activity in human temporal lobe epilepsy in vitro". Science 301(5632): 463; author reply 463.

31. Zhang, W., Yamawaki, R., Wen, X., Uhl, J., Diaz, J., Prince, D. A. and Buckmaster, P. S. (2009). Surviving hilar somatostatin interneurons enlarge, sprout axons, and form new synapses with granule cells in a mouse model of temporal lobe epilepsy. J Neurosci 29(45): 14247-14256. 